\title{
O.S.P.
}

\section{Projet professionnel et dynamique identitaire : Une approche socioconstructiviste}

Professional project and identity dynamics: A social constructivist approach

\section{Ghislain Mary et Anne-Marie Costalat-Founeau}

\section{(2) OpenEdition}

\section{Journals}

Édition électronique

URL : http://journals.openedition.org/osp/4259

DOI : 10.4000/osp.4259

ISSN : 2104-3795

Éditeur

Institut national d'étude du travail et d'orientation professionnelle (INETOP)

Édition imprimée

Date de publication : 7 mars 2014

ISSN : 0249-6739

Référence électronique

Ghislain Mary et Anne-Marie Costalat-Founeau, « Projet professionnel et dynamique identitaire : Une approche socioconstructiviste », L'orientation scolaire et professionnelle [En ligne], 43/1 | 2014, mis en ligne le 07 mars 2017, consulté le 19 avril 2019. URL : http://journals.openedition.org/osp/4259; DOI : $10.4000 /$ osp.4259

Ce document a été généré automatiquement le 19 avril 2019

(c) Tous droits réservés 


\title{
Projet professionnel et dynamique identitaire : Une approche socioconstructiviste
}

Professional project and identity dynamics: A social constructivist approach

\author{
Ghislain Mary et Anne-Marie Costalat-Founeau
}

\section{Introduction}

1 Avec la disparition du modèle de carrière de l'ère industrielle au profit des nouvelles formes de carrières sans frontière (Arthur \& Rousseau, 1996) et protéiformes (Hall, 1976, 2002, 2004), s'opère un glissement du collectif vers l'individuel dans la responsabilité de la fonction psychologique et sociale du travail. De nouvelles injonctions prennent forme que l'on pourrait résumer par «aide toi et le ciel t'aidera ». Ainsi le titre d'un article célèbre publié à la fin $\mathrm{du} \mathrm{xx}^{\mathrm{e}}$ siècle dans le champ de l'orientation professionnelle est «SelfEfficacy Training to Speed Reemployment: Helping People to Help Themselves » (Eden \& Aviram, 1993). L'objectif du conseil de carrière devient alors d'aider la personne à adopter une attitude plus " protéenne », définie par Hall comme la résultante d'un habile mélange de conscience de soi et d'adaptabilité, afin de transformer la perte d'un emploi - ou la saturation dans l'emploi actuel - en «bénédiction » (Zikic \& Klehe, 2006). Il est possible d'entrevoir, dans ce glissement progressif de la fonction du conseil, les nouvelles compétences qui sont attendues des conseillers au $\mathrm{xxI}^{\mathrm{e}}$ siècle. Faire coïncider les capacités et les valeurs avec une ou plusieurs profession(s) ne suffit plus, il faut dorénavant plus que jamais développer son empathie, son sens de l'écoute et trouver des recettes permettant de rendre les personnes aptes à gouverner leur vie de façon autonome. Des initiatives telles que celles générées au titre du paradigme du life design (Savickas et al. 2010) répondent à ce constat en focalisant l'attention sur l'augmentation de ce qu'ils nomment les "cinq C», caractéristiques de la faculté d'adaptation d'un individu (concern, control, curiosity, confidence, commitment). 
2 L'enjeu des choix de carrière engage l'individu dans une réflexion sur le sens qu'il souhaite donner à son parcours professionnel. Étant donné la prégnance de ce domaine d'activité dans l'organisation de la société et de nos vies, donner du sens à son parcours professionnel, c'est en même temps, au moins en partie, donner du sens à son existence. C'est donc tout naturellement que la thématique de l'identité attire l'attention de chercheurs (Dumora, Aisenson, Aisenson, Cohen-Scali \& Pouyaud, 2008) et de praticiens concernés par le renouvellement des méthodes d'accompagnement à la construction de projets personnels et professionnels. Tout en justifiant le recours aux histoires de vie comme technique d'exploration de l'identité, certains auteurs pointent le manque de méthodes qualitatives et idiographiques pour appréhender cette thématique dans la recherche comme dans la pratique (McMahon, 2008).

\section{Approche socioconstructiviste de l'identité et du projet}

\section{L'identité}

Une référence fréquemment mentionnée dans bon nombre de travaux sur le soi et l'identité reste la théorie esquissée par Mead, que l'on présente comme le fondateur de l'interactionnisme symbolique en psychologie sociale. La perspective qu'il a développée défend l'idée selon laquelle la conscience de soi n'est pas directement donnée au sujet mais s'acquiert très tôt à travers le développement du langage et l'utilisation de symboles préexistants à l'individu dans son environnement social. Il se rapproche en cela du constructivisme social de Vygotsky (1936). La conscience de soi dans l'interactionnisme symbolique de Mead est liée à la position occupée au sein de l'environnement social. Ainsi, chaque rôle dans la société est associé à un ensemble de symboles qui le définissent vis-à-vis des autres, dont découlent un point de vue particulier et certaines attentes comportementales. Les acteurs sociaux internalisent cette structure symbolique de manière active, ce qui signifie qu'ils ne se laissent pas passivement agir par les déterminants extérieurs liés à leur place dans la société. L'action socialement située joue un rôle primordial dans le développement de cette conscience individualisée, car c'est dans l'interaction avec autrui et avec les différents objets de connaissance du monde environnant que les individus doivent adapter leurs comportements et connaissances et que l'intégration se fait dès lors « active ». Pour rendre compte de cette complexité, Mead dichotomise le soi sous la forme d'un « Moi » objet de connaissance et d'un «Je » sujet agissant, vecteur d'autonomie et d'originalité. Soi est donc simultanément objet et sujet.

L'étude de la dynamique identitaire est celle d'une subjectivité agissante (Touraine, 1995) en constante interaction symbolique avec un environnement socioculturel duquel elle émerge et sur lequel elle agit en retour. Sur ces bases, le parallèle avec l'interactionnisme symbolique semble pouvoir s'établir. Cependant, il est une autre caractéristique de ce courant de pensée dont nous nous démarquons, il s'agit de la multiplication des sois. Nous nous rangeons en cela aux côtés de Bandura, lorsqu'il rejette l'idée d'un fractionnement de l'agentivité individuelle en une multiplicité de sois possibles :

«Une théorie de la personnalité prononcée en matière de sois multiples nous plonge dans de profondes eaux philosophiques. Cela requiert une régression des sois à la présidence d'un soi superviseur, qui sélectionne et organise le regroupement des sois afin de correspondre à des buts donnés. En réalité, il n'y a qu'un soi qui puisse visualiser différents futurs et sélectionner des modes d'action 
destinés à atteindre les futurs désirés et éviter ceux que l'on rejette » (Bandura, 1999, p. 15).

5 La dynamique identitaire telle que nous la définissons se rapproche donc de la définition de l'identité psychosociale formulée par Erikson (1972), qu'il décrit comme un long processus jalonné de crises, devant fournir un «sentiment subjectif et tonique d'une unité personnelle et d'une continuité temporelle» (1972, p. 13). Toutefois, si Erikson considérait que la construction identitaire se réalisait essentiellement pendant l'adolescence, pour se confirmer avec l'entrée dans l'âge adulte dans une identité achevée, cette problématique semble aujourd'hui relayée à tous les âges de la vie. Il faut donc retenir en priorité l'aspect processuel de l'identité mis en avant par Erikson. Cette focalisation sur l'aspect processuel nous confronte à un apparent paradoxe, puisque la raison d'être de ce processus est précisément de fournir à son hôte une identité, ce qui peut s'entendre comme un soi propre, saisissable. Or, pour mieux la cerner, il faut accepter de ne plus chercher à en saisir l'essence, afin d'en mieux -comprendre la dynamique. Une épistémologie constructiviste nous semble mieux à même de prendre en considération les aspects processuels et systémiques de la dynamique identitaire.

6 L'attention est donc ici portée sur les mécanismes de la subjectivité, lesquels ne sont ni considérés isolément ni, ce qui revient souvent au même, dans une simple interaction causale univoque dont on pourrait rendre compte par la démarche hypothéticodéductive. La règle est ici celle de la récursivité, ce qui signifie que tous les éléments du système (représentations de soi et d'autrui, catégories, référents prototypiques, attitudes et valeurs, motivations, capacités, épisodes autobiographiques) communiquent simultanément à plusieurs niveaux et s'influencent mutuellement. Les causalités sont donc multiples et opèrent par des chaînes complexes. Leur étude est rendue possible par la mise en évidence de tendances qui renforcent les liens entre certains éléments du système à l'instant où celui-ci est considéré dans sa globalité. Bien entendu, ces configurations identitaires n'ont de sens réellement que dans la continuité que suppose la dynamique du processus général (Ninot \& Costalat-Founeau, 2011). Sur ce point, bien que visant une certaine forme d'homéostasie, l'identité reste mue par l'adaptation et le changement, or, l'étude des évolutions temporelles des systèmes est rendue malaisée par la soudaineté et l'imprédictibilité des modifications significatives de leurs états. Toutefois, l'observation même ponctuelle de la dynamique identitaire nous renseigne déjà sur les stratégies identitaires subjectives (Camilleri et al., 1990) mises en place par l'individu pour s'adapter ou résister au changement.

\section{Le projet existentiel au sein de la dynamique identitaire}

7 L'enracinement philosophique d'une psychologie de l'identité liée au projet puise sa source dans l'existentialisme sartrien pour lequel l'homme n'est définissable que par ce qu'il choisit de devenir :

«L'homme n'est rien d'autre que ce qu'il se fait. Tel est le premier principe de l'existentialisme. C'est aussi ce qu'on appelle la subjectivité et que l'on nous reproche sous ce nom même. [...] L'homme est d'abord un projet qui se vit subjectivement » (Sartre, 1946).

Ajoutons à ce propos que la conception sartrienne de l'homme qui n'est que ce qu'il se destine à être renforce notre idée qu'il n'y a d'identité que celle que le sujet se construit :

«De la même façon, le pour-soi ne saurait être une personne, c'est-à-dire choisir les fins qu'il est, sans être homme, membre d'une collectivité nationale, d'une classe, 
d'une famille, etc. Mais ce sont des structures abstraites qu'il soutient et dépasse par son projet. [...] Mais ces caractéristiques n'ont pas de «Selbstständigkeit » [autonomie, ndla] : c'est avant tout son monde, c'est-à-dire illuminé par ses fins, qui se laisse découvrir comme Français, prolétarien, etc. » (Sartre, 1943, p. 568).

L'existentialisme athée prône la nécessité pour l'individu de s'engager dans le sens des convictions dont est porteur son projet et de défendre les valeurs auxquelles il choisit d'adhérer. Nécessité, le mot n'est pas exagéré puisque si l'être existentiel jouit de liberté dans ses choix, il ne choisit en revanche pas la liberté qui s'impose à lui. Refuser de choisir n'est pas une option, tout au plus une attitude de mauvaise foi pour ne pas avoir à assumer les conséquences de ses actes. Si donc Sartre fut précurseur en son temps, force est de constater que sa théorie existentialiste s'insère sans mal dans la pensée individualiste du xxI ${ }^{\mathrm{e}}$ siècle. Ceci étant, il en partage à la fois forces et faiblesses. Les valeurs suprêmes en sont la responsabilité et l'initiative. Or, ces dernières nous renvoient au malaise décrit par Herenberg (1998) des individus de l'ère postmoderne, incapables de se résigner à réaliser spontanément les choix qu'ils sont implicitement sommés d'assumer seuls et se réfugiant dans l'inaction et les pathologies ad hoc que sont la dépression et les dépendances. En outre, de par sa dénonciation du recours aux déterminismes comme justification de mauvaise foi des événements de vie, l'existentialisme comporte un risque de fragilisation de ce mécanisme de défense que peut être l'attribution externe. Dans le même temps, cette théorie se veut aussi porteuse d'espoir, celui de la possibilité d'une autodétermination du sujet, capable de relativiser l'importance qu'il accorde aux événements de vie qui l'affectent y compris et même plus particulièrement dans les crises, face à l'adversité.

\section{Constructivisme et identité dans le conseil en orientation}

Dans la pratique du conseil en orientation professionnelle, la perspective constructiviste met l'accent sur la production et l'organisation du sens de leur vie, de leurs actions et de leur environnement par les individus eux-mêmes. Cette orientation épistémique fournit donc un cadre conceptuel pour apprécier qualitativement les nouveaux modes d'adaptation au travail et de construction de carrière dans un contexte postmoderne où l'aptitude à surmonter les crises et les transitions, qui appellent une remise en question profonde de l'identité, devient une responsabilité à part entière de l'individu.

11 Guichard $(2004,2008)$ a proposé un modèle d'entretien constructiviste de conseil en orientation destiné aux jeunes dont l'objectif est de parvenir à les rendre plus autonomes et libres dans la définition de leurs projets professionnels. Les «formes identitaires subjectives » qu'il se propose d'investiguer englobent « un ensemble de manières d'être, d'agir et d'interagir en lien avec une certaine représentation de soi dans un certain contexte ». Il part du constat que l'identité se construit dans un système social structuré proposant une certaine "offre identitaire " composée de catégories sociales variées et plus ou moins prégnantes, auxquelles correspondent des modes de rapport à soi, des manières de se percevoir, de se penser et de se construire au sein de son environnement socioculturel. Ceci étant, l'individu n'est pas passivement déterminé par l'offre identitaire de la structure sociale, il se l'approprie, il la fait sienne autant qu'il se fait soi. Le modèle «se faire soi » élaboré par cet auteur vise donc à rendre compte de l'élaboration subjective dont est l'objet cette offre identitaire. 
12 L'épistémologie constructiviste et la théorie des systèmes ont de nombreux points en commun, parmi lesquels figure en bonne place l'axiome ou le principe téléologique. Celuici pose que la production de connaissances est organisée par les finalités du sujet connaissant. En découle que l'étude de sa subjectivité ne consiste plus à en élucider les causes probables mais à en identifier les fins plausibles. Cette perspective nous amène à reconsidérer la place du projet de vie dans la dynamique identitaire comme un possible organisateur des percepts, des affects et des représentations qui la composent. L'axiome phénoménologique du constructivisme pose à son tour que le sujet connaissant n'est pas distinct de l'objet à connaître. Le réel n'est pas donné, il est construit par le sujet en fonction d'un rapport spécifique à l'objet et d'une organisation préalable de ses connaissances. Ainsi que l'a formulé Piaget (1936, p. 312), « l'intelligence [...] organise le monde en s'organisant elle-même ». Ainsi connaître le monde revient à se construire soimême. Cette intrication propre au développement de l'intelligence se trouve renforcée par la problématique de l'identité puisque le sujet connaissant devient son propre objet de connaissance. En outre, il devient compliqué de continuer à considérer le sujet comme un objet de connaissance classique à la manière de certaines théories cognitivistes sur le soi.

Dans la théorie des systèmes vivants ou living systems framework (LSF) (Ford, M.E. \& Ford, D.H., 1987), l'individu psychologique est conceptualisé comme un être agissant par le truchement de son monde expérientiel, qui n'est autre qu'une construction mentale du monde extérieur internalisée à travers l'expérience vécue. Ce concept de système vivant peut éclairer la manière dont fonctionne l'environnement intérieur de l'identité psychosociale dont fait mention Erikson (1972), démarche déjà entreprise par Zavalloni et Louis-Guérin (1984) lorsqu'elles développèrent la méthode IMIS que nous avons adaptée aux besoins du conseil de carrière et qui sera décrite plus loin. Patton et McMahon (1997, 2006) ont pour leur part identifié une série de composantes essentielles de la théorie des systèmes qu'elles ont pu intégrer à leur modèle du conseil de carrière. Cette approche tisse des liens entre la théorie, l'expérimentation et la pratique, exigeant des conseillers un effort pour s'extraire de la posture d'expertise basée sur des causalités linéaires, afin d'apprendre à interpréter les causalités multiples et circulaires au sein desquelles le sujet construit son expérience de l'environnement. La transposition de ce paradigme de la complexité aux problématiques de l'orientation professionnelle, de la construction de carrière et du projet de vie est encore récente, appelant à en formaliser les méthodes qui permettront d'élaborer des projets en s'appuyant sur cette subjectivité interactionnelle et agissante.

Dans la redéfinition des rapports entre chercheur-observateur et sujet-observé se trouve une des raisons fondamentales du recours aux constructivismes pour l'étude des systèmes complexes tels que le sont en psychologie l'identité et le projet. Selon Patton et McMahon (2006), la relation de conseil établie entre le consultant et le conseiller peut aussi être considérée comme un système à part entière. Le conseiller devient un élément du système d'influences sur le développement de carrière de l'individu. Dans le même temps, l'individu devient un élément du système d'influences sur le conseiller. Dans cette interaction systémique, tous deux utilisent le langage pour coconstruire un projet donnant du sens à la carrière. Lemoine (2011, p. 72) étend ce constat d'interdépendance à la recherche sur le bilan de compétences et ce faisant il dénonce «le schéma classique d'une science de l'objet qui n'intègre pas l'idée que le sujet étudié est humain, de même nature que le scientifique, et à ce titre capable de saisir, de comprendre et de s'approprier 
aussi des informations, qui de plus viennent de lui et sont donc censées lui revenir ». Lemoine $(2007,2011)$ préconise donc le recours à une démarche constructiviste tout en s'interrogeant sur la possibilité d'obtenir des informations valides par ce procédé qui consiste à associer le sujet à la recherche qui est menée sur lui :

«À ce sujet il faut rappeler que l'objectivité n'est pas une dimension donnée d'emblée. Elle est une construction à partir de critères formels qu'il faut définir et partager avec une communauté scientifique. Il s'agit ici de la réaliser avec le concours de l'intéressé qui participe alors à cette construction. Le chercheur n'en a pas l'exclusivité, n'est pas objectif en soi, et n'est jamais en dehors du champ social étudié »(Lemoine, 2011, p. 75).

\section{L'egoécologie}

15 Aborder la construction du projet sous l'angle de la dynamique identitaire du sujet suppose d'investiguer avec lui l'univers de significations qu'il a construit sur la base de ses expériences, ce que Zavalloni $(1984,2007)$ a appelé l'environnement intérieur opératoire (EIO) de l'individu en référence à Erikson (1972). Celui-ci, de nature représentationnelle, cognitive et affective, n'est pas une simple collection de catégories, d'images et de traits. Il est l'instrument de la dynamique identitaire par lequel le sujet perçoit son environnement et interagit en son sein, tout en satisfaisant aux aspirations d'unicité, d'unité et de valorisation dont est garante l'identité. Nous postulons que toutes les composantes du projet s'y trouvent (Mary \& Costalat-Founeau, 2012), y compris si celui-ci n'a pas encore germé dans une forme cohérente et tendue vers un but intentionnel. Le recours à une méthodologie constructiviste doit justement permettre de coconstruire avec le sujet ce projet dont nous souhaitons étudier les relations avec la dynamique identitaire. Dans la perspective egoécologique, les unités représentationnelles, produites pour définir les groupes sociaux stimuli qui forment la matrice sociale du sujet, sont considérées comme des données du premier degré, c'est-àdire dénuées de contexte, appelant un travail plus approfondi d'explicitation. L'intérêt ne sera donc pas tant porté sur ces données du premier degré mais bien plutôt sur les interactions dynamiques qu'entretiennent entre elles ces représentations par l'intermédiaire des significations, des expériences, des valeurs et des émotions dont elles sont porteuses.

16 À l'instar de Vygotsky (1986), l'egoécologie considère donc le mot comme une unité d'analyse ouvrant sur un univers de symboles, d'expériences concrètes et d'émotions, qu'elle nomme "pensée de fond». L'IMIS (investigateur multistade de l'identité sociale) vise à faire émerger cette pensée de fond qui accompagne le mot à la périphérie de la conscience et donne au sujet une impression de réalité vécue sur son discours. Déployer le contexte sous-jacent au mot permet d'entrevoir les mécanismes à travers lesquels il prend du sens et en donne en retour. Le mot apparaît comme un instrument de transaction véhiculant du sens symbolique non seulement au sein de l'environnement intérieur mais également entre le sujet et son environnement socioculturel. Tout mot qui révèle lors de cette analyse un particulièrement vaste réseau d'associations apparaît comme le centre d'un "circuit affectif-représentationnel », composé de représentations liées à des affects. Ceci permet d'éclairer son importance dans le processus de l'identité psychosociale, raison pour laquelle il peut alors être qualifié de «mot identitaire». En partant des mots ou unités représentationnelles que le sujet utilise pour définir les groupes sociaux stimuli qui composent la matrice sociale objective de son identité, il est 
donc possible à condition de ne pas en rester au niveau de ces données de premier degré, d'investiguer et de décrire la structure de significations et d'émotions qui reflète le fonctionnement de sa subjectivité et la manière dont se construit son identité. Notre objectif en recourant à cette méthode était de montrer qu'elle permettrait aussi de mettre en évidence la construction du projet, en raison des liens étroits qu'entretiennent l'identité et le projet, professionnel et existentiel. Cette approche socioconstructiviste a donc été choisie pour analyser, in fine, les interactions entre la construction du projet et celle de l'identité.

\section{Méthode d'exploration de l'identité et du projet}

\section{L'IMIS}

17 L'investigateur multistade de l'identité sociale (IMIS) a été inventé par Zavalloni et LouisGuérin (1984) pour explorer à travers une approche idiographique les dimensions symboliques, affectives, évaluatives et motivationnelles, des mouvements d'identification et de différenciation qui composent l'identité psychosociale. La passation de l'IMIS se déroule en trois phases.

18 La première étape est la phase de recueil du répertoire sémantique de l'identité sociale. Elle vise à recueillir des unités représentationnelles (UR) qui sont les descriptions que donne le sujet, sous la forme de cinq mots ou courtes phrases, des groupes sociaux stimuli qui lui sont présentés. Le choix des groupes stimuli en amont de la passation de ce questionnaire est déterminant pour l'efficacité de la méthode, et il est conseillé d'y faire participer le sujet afin que les groupes retenus coïncident autant que possible simultanément avec la problématique du chercheur et l'identité psychosociale du sujet. Dans notre cas, nous avions choisi d'investiguer les représentations des groupes sociaux correspondant aux activités professionnelles et extraprofessionnelles dans lesquelles chaque sujet s'était impliqué et qu'il jugeait importantes dans son parcours. Les mots et courtes phrases recueillis à cette étape feront l'objet d'approfondissements successifs tout au long des phases ultérieures du protocole de l'IMIS.

19 Au cours de la deuxième phase, il est demandé au sujet de positionner chacune de ses réponses sur deux échelles. La première exprime le degré d'applicabilité à soi de chaque unité représentationnelle produite. Ceci permet entre autres de repérer si le sujet s'identifie aux groupes stimuli. La seconde échelle spécifie la valeur, positive ou négative, de chaque unité représentationnelle produite. L'espace élémentaire de l'identité sociale (EEIS) est l'aboutissement de cette seconde phase de l'IMIS. Il présente une structure topologique des représentations identitaires produites composée de quatre champs distincts délimités par l'intersection des deux axes soi/non-soi et positif/négatif (voir fig. 1). 
Fig. 1. Espace élémentaire de l'identité sociale (EEIS)

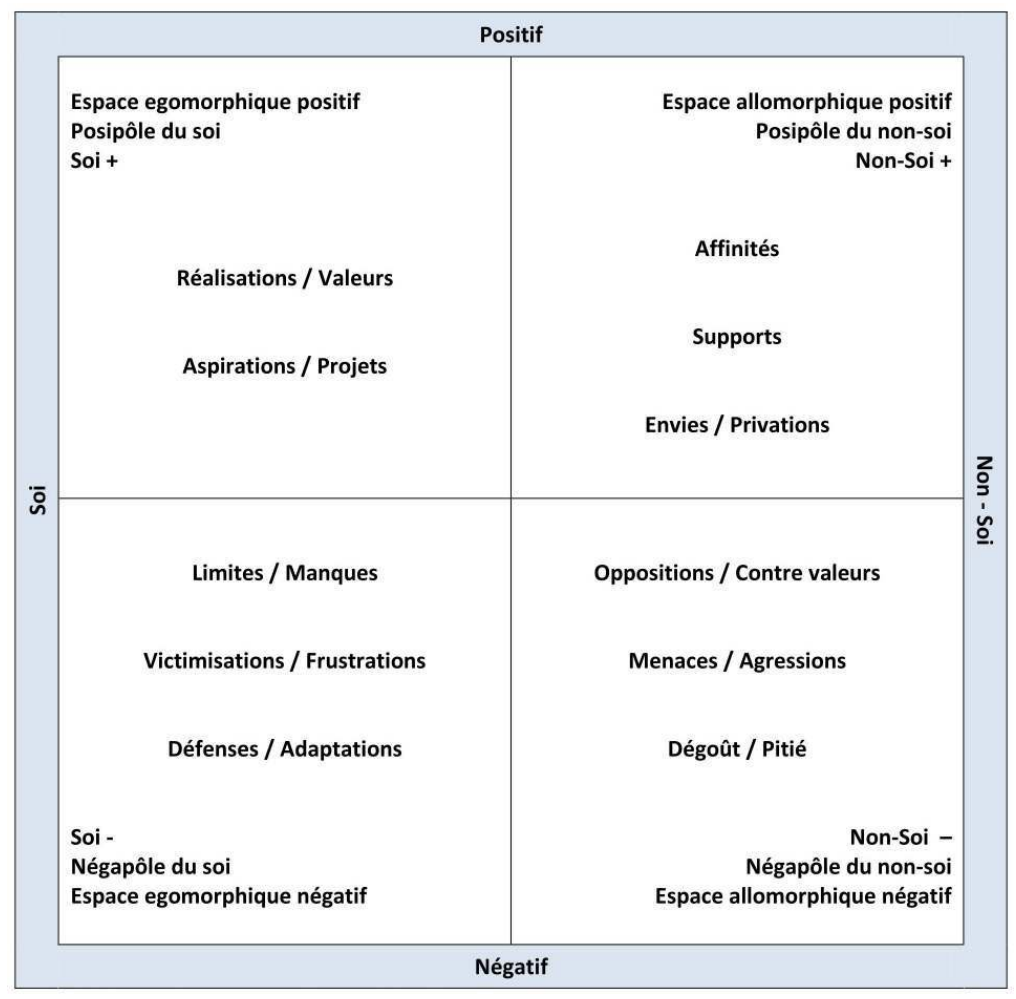

Fig. 1. Elementary space of social identity

L'espace du soi positif renferme les représentations positivement chargées, les réalisations, les aspirations et les valeurs vers lesquelles tend le sujet. On peut y lire en filigrane ses projets de vie :

«Ici le soi vit dans l'assentiment et dans l'acceptation de soi, la fierté, le désir, les préférences et en communion avec son groupe. Se dessine aussi le contour du monde tel que l'on souhaiterait qu'il soit » (Zavalloni, 2007, p. 49).

Le soi négatif comporte les limites personnelles à l'épanouissement du sujet, ses faiblesses comme par exemple l'incompétence, la victimisation dont il se sent l'objet mais aussi des défenses et différents modes d'adaptation élaborés pour réagir à ses difficultés, au stress ressenti ou aux menaces et agressions extérieures.

Le non-soi négatif rassemble les contre-valeurs auxquelles le sujet s'oppose, ce qu'il ne veut pas être ou qu'il ne faut pas faire, ainsi que les menaces et agressions telles que les discriminations, l'exploitation, les violences et, plus généralement, ce qu'il rejette, non seulement par dégoût mais aussi quelques fois par pitié.

L'espace allomorphique positif ou négapôle du non-soi se constitue de supports émotionnels, symboliques et instrumentaux permettant une collaboration positive et des échanges mais également d'objets de désirs valorisés mais non atteints, faute de capacités, de ressources ou par privation.

Le troisième stade est le moment de l'analyse du réseau associatif, qui se conduit comme un entretien semi-directif d'une sorte particulière, que Zavalloni (1971) a baptisé "méthode d'introspection focalisée ». Il est structuré autour d'une série de questions qui allient la précision de leurs objectifs à la liberté qui est laissée au sujet de développer à travers ses réponses les expériences vécues et les thèmes qui lui semblent pertinents pour 
l'explicitation de ce à quoi fait référence le mot identitaire dans son propre système de valeurs et de significations. Les questions successives correspondent toutes à une partie bien définie de l'élucidation du sens subjectif de l'unité représentationnelle. La lecture du guide d'entretien que nous avons adapté à la thématique du conseil de carrière pourra éclairer le lecteur sur la nature de ces approfondissements (voir fig. 2).

Figure 2. Guide d'entretien de la phase 3 de l'IMIS, adapté à notre problématique

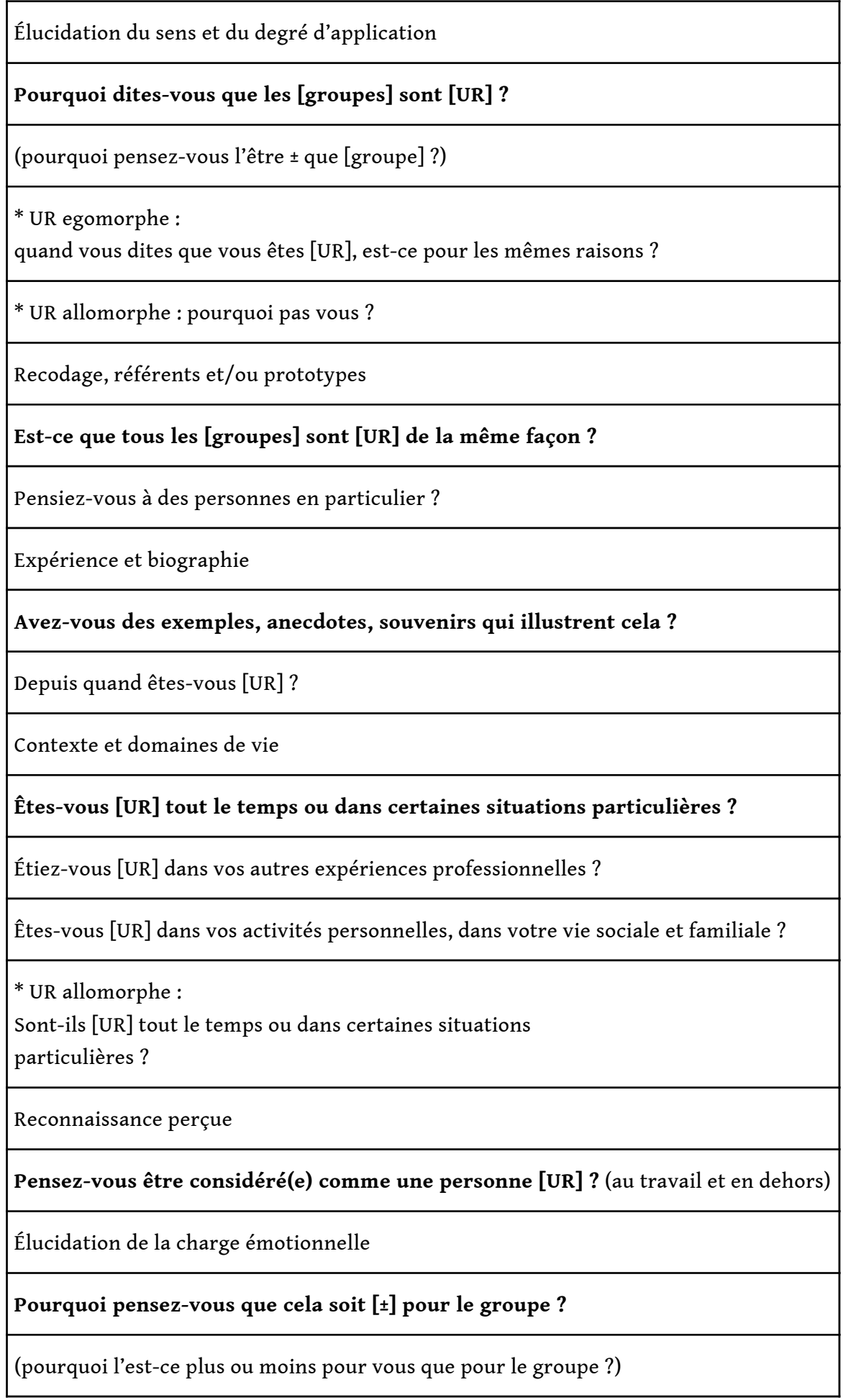


* UR egomorphe : pourquoi est-ce $[ \pm]$ pour vous?

* UR allomorphe : pourquoi serait-ce [ $[$ ] pour vous?

Fig. 2. MISI phase 3 interview guide, adapted to our research

\section{Participants}

Cinq sujets prirent part à nos investigations en acceptant d'apporter leurs réponses aux trois phases de notre protocole adapté de l'investigateur multistade de l'identité sociale (IMIS) de Zavalloni et Louis-Guérin (1984). Nous nommerons ces sujets François, Will, Élyse, Jean et Luc. Tous étaient des salariés engagés dans une prestation de bilan de compétences afin de préparer une évolution ou une reconversion professionnelle, à l'exception de François qui était demandeur d'emploi.

\section{Présentation et analyse des données de l'exploration egoécologique}

26 À l'issue de la troisième phase de l'IMIS, nous disposons de suffisamment de matériel discursif pour éclairer le sens subjectif de chaque unité représentationnelle produite lors de la première phase pour décrire des groupes sociaux stimuli. Nous pouvons donc faire émerger les relations qu'entretiennent entre elles ces unités représentationnelles, qui structurent la dynamique identitaire, sous la forme de ce que Zavalloni et Louis-Guérin (1984) ont nommé le « noyau dynamique sociomotivationnel » (voir fig. 3). Rapportées ici, ces observations pour chacun de nos sujets seraient trop longues, nous présenterons donc à titre d'exemple le cas d'Élyse, afin d'illustrer succinctement comment se présente l'analyse de la dynamique identitaire explorée au moyen de l'IMIS. Ajoutons avant de procéder à cet exposé que nous recommandons d'associer le sujet à cette ultime phase d'analyse, notamment lorsqu'elle s'inscrit dans le processus d'un accompagnement à la construction du projet professionnel, dans un souci de cohérence avec ce que nous avons précédemment exposé concernant l'approche constructiviste et conformément aux récentes recommandations de Zavalloni (2007).

\section{Le cas d'Élyse}

Élyse se définit elle-même comme une assistante polyvalente, ce qui n'apparaît pas sur ses contrats de travail mais retranscrit mieux la diversité de ses missions. Son parcours professionnel est constitué d'expériences professionnelles successives n'excédant généralement pas une durée de deux ans. Ces changements d'emplois fréquents ne sont pas subis mais voulus. Élyse a jusqu'à présent fait évoluer sa carrière en naviguant entre des emplois à forte composante administrative de relativement courte durée, ce qui lui permettait d'acquérir de nouvelles connaissances et compétences dans chaque nouvelle entreprise. Elle n'aime pas la routine qui s'installe une fois maitrisées les tâches qui composent son activité professionnelle. Son entrée dans la prestation d'accompagnement au projet est justifiée selon ses propres dires par un mécontentement dans l'emploi d'assistante commerciale qu'elle occupe alors. Deux principales raisons sont de prime 
abord évoquées, un ennui lié au sentiment de plafonnement dans son activité qu'elle maîtrise maintenant et un manque de reconnaissance qu'elle reproche à sa hiérarchie qui ne sait lui adresser « que des reproches » quant à son travail.

Dans le cas d'Élyse les groupes stimuli, choisis avec le sujet de manière à refléter ses activités passées les plus significatives, étaient les suivants: assistantes polyvalentes, assistantes de communication bilingues, chargées de recouvrement. Nous avons ainsi regroupé les unités représentationnelles produites par Élyse pour définir chaque groupe stimulus en un certain nombre de thématiques très prégnantes dans son discours (fig. 3). Les différentes unités représentationnelles qui composent un même thème entretiennent entre elles des relations de contiguïté et de spécification réciproque. Dans le vocabulaire de l'IMIS, cela signifie que leurs sens sont proches et/ou se complètent. Par exemple au sein de la thématique de la polyvalence, les assistantes polyvalentes sont "multitâches " et «adaptables » parce qu'elles " apprennent facilement » et qu'elles sont «curieuses ». Toutes ces caractéristiques sont en outre des composantes du soi positif d'Élyse, indiquant des capacités et valeurs qu'elle fait siennes en même temps qu'elle les attribue au groupe. Deux autres thèmes complètent le soi positif d'Élyse, celui de l'ouverture et celui de la rectitude. Ce dernier possède la caractéristique peu commune d'être également représenté dans le soi et le non-soi négatifs, marquant ainsi la forte ambivalence des capacités et valeurs qui le composent. Sur le plan motivationnel, cette configuration joue un rôle important dans la construction du projet. Elle témoigne d'une tension se jouant à la fois dans le rapport à soi et vis-à-vis d'autrui, motivant Élyse à faire des choix qui lui permettront d'atténuer cette tension. Afin de mieux comprendre ce à quoi renvoie cette ambivalence, il est nécessaire de revenir sur les parties du discours d'Élyse qui éclairent les significations attribuées à ces unités représentationnelles. Élyse nous y explique que sa carrière n'a jusqu'à présent été composée que d'une multiplication de courtes expériences à divers postes d'assistanat administratif. Le type d'emploi dans lequel sont principalement valorisées les capacités liées au thème de la rectitude d'Élyse. Cette dernière a donc développé de son mieux ces capacités correspondant à son rôle social, d'autant plus aisément qu'elle admet être naturellement "carrée ", caractéristique qu'elle tiendrait de sa mère "encore plus carrée, trop même ». Dans la personne de la mère d'Élyse apparaît donc ce que l'egoécologie désigne comme un "prototype identitaire ", personne réelle au contact de laquelle a été internalisée une caractéristique essentielle de la dynamique identitaire. Le soi, le groupe et le prototype identitaire constituent ensemble la «triade identitaire " que l'on retrouve souvent à l'origine de l'intériorisation d'une caractéristique essentielle de soi cristallisée dans un «mot identitaire ». De fait, à travers le discours d'Élyse, nous avons identifié l'unité représentationnelle "carrées » comme étant un mot identitaire, c'est-à-dire un terme renvoyant à un vaste réseau affectivo-cognitif d'épisodes autobiographiques, de valeurs, de motivations et de définitions de soi et du monde. Ceci a permis de mettre en évidence son importance dans la dynamique identitaire et le projet d'Élyse. Maintenant, ce mot identitaire "carrées", en étant à la fois positif et négatif, exprime en même temps le revers de l'aptitude ambivalente à la rectitude. Dans son soi négatif, le mot identitaire « carrées » est accompagné de l'unité représentationnelle « inflexibles » qui en complète le sens. La jeune femme explique que le fait d'être carrée, bien qu'étant une capacité reconnue dans le travail administratif, peut parfois s'avérer être un handicap et un frein au développement d'autres capacités utiles dans les relations humaines. Elle en est consciente, poussé à l'extrême, le thème de la rectitude devient une composante du non- 
soi négatif d'élyse, dont le rejet est exprimé à travers les unités représentationnelles " sans scrupule » et « robotisées ".

De ce constat d'ambivalence découle que le thème de la rectitude recèle des qualités d'Élyse dont les revers bloquent le développement d'autres qualités, capacités et valeurs auxquelles elle aspire. Ces relations d'opposition antithétique sont retranscrites dans la synthèse du noyau socio-dynamique motivationnel d'Élyse (fig. 3) entre la rectitude d'une part et les thèmes de l'ouverture et de la créativité d'autre part. En conséquence, l'une des demandes d'Élyse auxquelles doit répondre son projet en cours de construction est de nécessiter moins de rectitude tout en permettant de développer l'ouverture et la créativité. Fort heureusement, l'exploration du sens subjectif des unités représentationnelles qui composent ces deux thèmes a pu mettre en évidence des aptitudes et dispositions favorables d'Élyse envers celles-ci qui ont été jusqu'à présent brimées par les attentes sociales des situations professionnelles dans lesquelles elle a évolué. Élyse, s'étant identifiée elle-même comme une personne "carrée » en s'identifiant à des groupes socioprofessionnels définis comme tels, s'était dans le même temps définie comme non créative. Son nouveau projet en construction s'accompagne donc de nécessaires redéfinitions de soi et d'identifications nouvelles à explorer, travail amorcé par l'analyse de sa dynamique identitaire rendue possible par l'IMIS.

En outre, il y a un autre point sur lequel son projet professionnel, jusqu'à présent guidé par l'acquisition d'une multitude de savoirs et compétences utilisables qui la font aujourd'hui se définir comme une "assistante polyvalente", est en train d'être transformé. Les valeurs professionnelles d'Élyse évoluent à un tournant de sa carrière. Elle souhaite désormais que sa mobilité professionnelle ne soit plus simplement horizontale mais prenne un tournant vertical, afin de gravir quelques échelons dans l'échelle sociale. D'un projet de développement «autodidacte » de sa polyvalence, elle souhaite désormais passer à une spécialisation plus approfondie devant déboucher sur une meilleure reconnaissance et plus de responsabilités. Cette évolution se fait en parallèle d'une recherche de stabilisation dans sa vie personnelle. Dans la synthèse de son noyau socio-dynamique motivationnel, cela se traduit par la présence du thème de l'utilité dans l'espace du non-soi positif, qui signifie qu'Élyse ne se sent plus utile en tant qu'assistante polyvalente. Le thème de l'utilité auquel elle aspire néanmoins se trouve dans une relation de support vis-à-vis des unités représentationnelles « mal payées » (soi négatif) et « pas reconnues » (non-soi négatif). 
Image 200001 A900002C720000412E5ACBD756.wmfFigure 3. Noyau socio-dynamique motivationnel d'Élyse

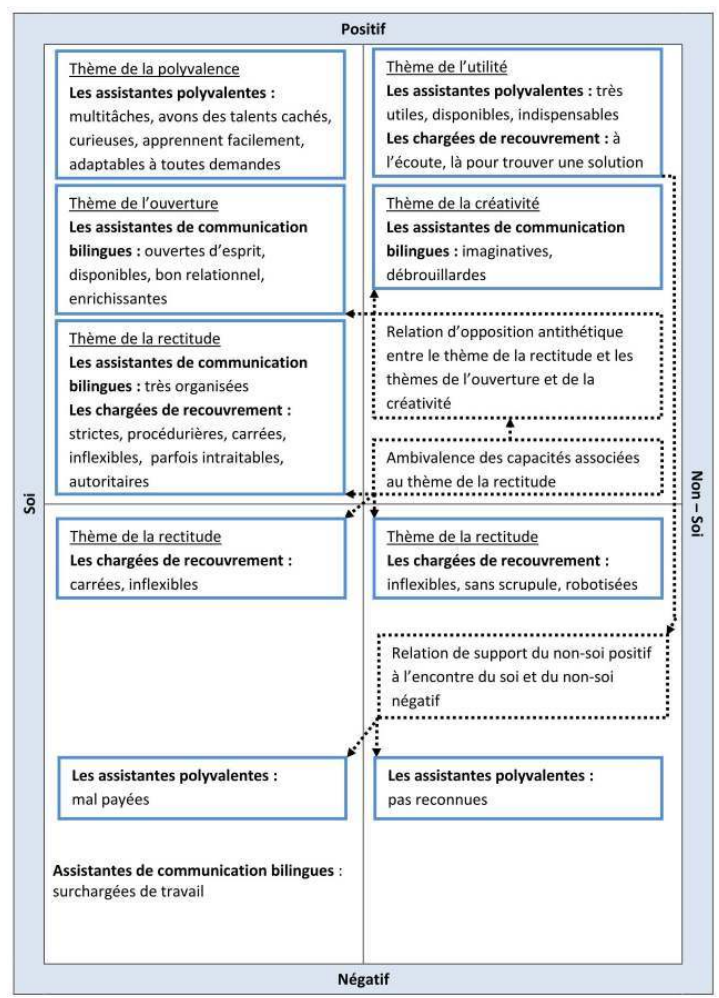

Figure 3. Elyse's motivational socio-dynamic core

\section{Discussion sur les liens entre identité et projet}

L'exemple présenté illustre bien la manière dont l'intériorisation active de l'environnement social sert de base à la construction de l'identité dans une interaction, déterminant en partie les projets et étant déterminée en retour selon le principe de la récursivité. La construction de nouveaux projets implique donc une remise en question de l'identité, à travers le sens donné à nos actions, ainsi que nos modes de rapports aux autres et à soi.

Nous avons donc pris le parti d'analyser la dynamique identitaire de manière à faire ressortir son implication dans l'émergence du projet. Il nous faut ajouter à cette grille d'analyse deux composantes indissociables du projet, comme de l'identité, que sont l'action et la socialisation. Une théorisation des liens entre ces notions est déjà esquissée dans le paradigme socioconstructiviste de l'action en contexte proposé par Young, Valach et Collin (Young \& Valach, 1996, 2004, 2006; Young \& Colin, 2004), suggérant que le processus de carrière puisse être considéré comme un système orienté vers un but, dans lequel l'action représente l'unité à court terme, le projet l'unité à moyen terme et la carrière l'unité à long terme de processus intentionnels dans des domaines variés. Le modèle théorique du système capacitaire que nous développons (Costalat-Founeau, 1997, 2008) définit quant à lui l'action comme un projet à temporalité moyenne, ce qui signifie qu'elle est située au cœur même du projet et non simplement conséquence de ce dernier. Ce modèle insiste aussi sur le caractère social de l'action en contexte qui tout comme le 
projet se formule et s'argumente sur la base d'expériences, d'aspirations et de ressources qui renvoient à l'environnement social du sujet.

La méthode d'investigation retenue a permis d'éclairer la double tension, à la fois interne (rapport à soi) et externe (rapport à autrui), dans laquelle émerge le projet en devenir. L'idée d'une tension interne renvoie au paradoxe de l'identité qui est garante du "sentiment subjectif et tonique d'une unité personnelle et d'une continuité temporelle " (Erikson, 1972, p. 13), en même temps qu'elle permet nécessairement à l'individu de s'adapter et d'évoluer. Un passage du discours d'un autre de nos sujets, Will, illustre bien cette tension dans le rapport à soi :

«Vous savez, c'est un peu comme les montagnes russes hein, il y a des moments où je suis en haut et il y a des moments où je suis en bas mais ça c'est le caractère de chacun. Le mien est bien spécifique. Il y a des moments où je suis très haut et je m'y plais mais il $\mathrm{y}$ a des moments où je m'ennuie. Il faut que je redescende un peu. J'ai besoin de cette excitation qui est dans le négatif mais j'en ai besoin. Je vais me brûler mais je me relève. Je crois que j'ai besoin de ça. C'est en moi quoi. »

Les personnes qui s'engagent dans une redéfinition de leurs projets personnels et professionnels amorcent en même temps une transition dans leur identité psychosociale. Celle-ci est aussi rapport à autrui, comme le sont également l'action et le projet. L'environnement social influence les projets des individus qui doivent se déterminer en tenant compte des projets d'alter dans lesquels ils sont inclus. Parmi nos sujets, François est celui qui exprime cette tension le plus consciemment : «La sensation qu'on décide à votre place ce qui est bien pour vous, ça c'est assez... C'est pénible.» L'interactionnisme symbolique (Mead \& Morris, 1934) ou encore la notion d'habitus (Bourdieu, 1980) vont jusqu'à postuler le primat de la société sur la détermination du sujet. À l'inverse, la philosophie existentialiste (Sartre, 1943, 1946) invite à considérer le projet comme une émancipation et un principe orientant la connaissance de soi, d'autrui et du monde. Une perspective socioconstructiviste doit permettre de rendre compte en même temps de l'importance des caractéristiques individuelles et de l'environnement social dans la dynamique identitaire subjective, ceci, afin de permettre aux individus de saisir ce qu'impliquent ces deux sources dans la construction du projet et d'y puiser les ressources nécessaires pour se construire un projet.

L'action est un support à la concrétisation expérientielle et à la validation sociale des représentations de soi, notamment en ce qui concerne les capacités et les valeurs (Costalat-Founeau, 1999) auxquelles on ne peut manquer de prêter attention lors de la construction du projet. La reconnaissance d'autrui, ainsi que de soi par soi, se gagne sur ce terrain. Plus encore, l'exemple d'Élyse montre bien comment le sens et la valeur mêmes des capacités déployées dépendent du contexte de l'action. Plébiscitée dans le travail administratif, la rectitude peut s'avérer handicapante dans les relations humaines pour l'ouverture aux autres ou la créativité. D'où l'importance de bien faire coïncider ses valeurs avec le contexte vers lequel on souhaite s'orienter. Trois types de reconnaissance professionnelle ont été mis en avant au cours de nos entretiens. Celle des capacités tout d'abord dont il a été question à travers l'exemple d'Élyse mais qui bien entendu est importante pour tous. Celle des valeurs ensuite qui peuvent poser problème lorsqu'elles ne sont pas partagées par l'entourage professionnel et/ou l'organisation. Le troisième type de reconnaissance qui a été spontanément mis en avant par tous nos sujets est celui de l'utilité du travail fourni. Il y a visiblement là un besoin fondamental à satisfaire ainsi que le clame déjà Dejours (2003) dans le champ de la psychodynamique du travail. Il apparaît enfin qu'à travers l'action socialement située se coconstruisent de manière 
intersubjective les identités professionnelles, ainsi que nous le suggère fortement François lorsqu'il explique que certaines des unités représentationnelles utilisées pour décrire ses groupes sociaux stimuli sont en réalité des sentiments collectivement partagés et des représentations construites dans l'interaction : «C'est pour ça que le " nous " là est important parce que c'était un vrai travail d'équipe et ces sentiments, c'est des choses qu'on partageait entre nous. »

\section{Conclusion}

Les investigations menées au moyen de l'IMIS nous ont permis d'appréhender efficacement à travers le discours des sujets les interactions entre le projet, l'action et la socialisation, qui forment au sein de la dynamique identitaire une boucle systémique pouvant s'avérer parfois vertueuse. Le modèle théorique du système capacitaire (CostalatFouneau, 1997, 2008) donne à la capacité le rôle d'activateur des phases identitaires. Dans les situations favorisant la congruence de la capacité subjective construite avec la capacité objective ou normative perçue, le sujet se trouve en phase d'acuité représentationnelle alors qu'à l'inverse l'incongruence de ces deux vecteurs d'actualisation de la capacité instillera des phases de diffusion représentationnelle. L'acuité est synonyme d'une meilleure représentation de soi dans son environnement, compatible selon nous avec la vision de Larson (2000), d'une attention accrue focalisée sur le champ de l'expérience ayant pour effet de faire émerger de sa complexité une perception organisée. Ces phases positives correspondent à des moments où le sujet est mieux prédisposé à l'action. La diffusion implique à l'inverse que l'action du sujet est inhibée et que sa faculté de représentation est moins efficace. Lors de ces phases, il peut plus facilement se laisser submerger par l'émotion qui ne joue alors plus son rôle de maintien énergétique de l'attention. Le sujet a besoin de prendre du recul afin de trouver une stratégie lui permettant de résoudre l'incongruence de son système capacitaire. Une proportion relativement importante des individus recherchant une aide dans une phase de transition professionnelle nous semble rencontrer une problématique similaire sur le plan des capacités qu'ils pensent pouvoir mettre en œuvre pour mener efficacement leur évolution de carrière. L'approche constructiviste de la dynamique identitaire suggère que les capacités dépendent à la fois du contexte social et de l'histoire de vie du sujet. Certaines représentations et capacités associées aux expériences passées deviennent inaccessibles, parfois même inappropriées, lorsque l'individu change de contexte, tandis que d'autres peuvent être transférées et mises en œuvre pour surmonter les difficultés. La construction du projet doit permettre de retrouver la cohérence subjective entre l'individu, son histoire et son environnement. Au regard de ces observations, le recours à une méthodologie idiographique nous semble plus prometteur que l'instrumentation nomothétique classique. Toutefois, les meilleurs résultats seront à n'en pas douter obtenus avec un mélange habile d'approches positivistes et constructivistes (Bernaud, Danet \& Dinar, 2009 ; Cohen-Scali \& Kokosowski, 2010). 


\section{BIBLIOGRAPHIE}

Arthur, M.B. \& Rousseau, D.M. (1996). The boundaryless career: A new employment principle for a new organizational era. New York: Oxford University Press.

Bandura, A. (1999). A social cognitive theory of personality. In L. Pervin \& O. John (Eds.), Handbook of personality (pp. 154-196). New York: Guilford Publications.

Bernaud, J.L., Danet, L. \& Dinar, M. (2009). Comparaison des effets de trois modes de restitution de questionnaires d'intérêts : nomothétique, constructiviste et intégré. L'orientation scolaire et professionnelle, 38(2), 135-160.

Bourdieu, P. (1980). Le sens pratique. Paris : Minuit.

Camilleri, C., Kastersztein, J., Lipiansky, E.M., Malewska-Peyre, H., Taboada-Leonetti, I. \& Vasquez, A. (1990). Stratégies identitaires. Paris : Presses Universitaires de France.

Cohen-Scali, v. \& Kokosowski, A. (2010). La question de l'opérationnalisation du modèle de la construction de sa vie : Commentaire de l'article collectif « Construire sa vie (Life designing) : un paradigme pour l'orientation au XxI siècle ». L'orientation scolaire et professionnelle, 39(1), 87-99.

Costalat-Founeau, A.M. (1997). Identité sociale et dynamique représentationnelle. Rennes : Presses Universitaires.

Costalat-Founeau, A.M. (1999). Identity dynamic, action and context. Journal for the theory of social behaviour, 29, 289-300.

Costalat-Founeau, A.M. (2008). Identité, action et subjectivité : le sentiment de capacité comme un régulateur des phases identitaires. Connexions, 89, 63-74.

Dejours, C. (2003). L'évaluation du travail à l'épreuve du réel : Critique des fondements de l'évaluation. Paris : INRA.

Dumora, B., Aisenson, D., Aisenson, G., Cohen-Scali, v. \& Pouyaud, J. (2008). Les perspectives contextuelles de l'identité. L'orientation scolaire et professionnelle, 37(3), 387-411.

Eden, D. \& Aviram, A. (1993). Self efficacy training to speed reemployment: helping people to help themselves. Journal of Applied Psychology, 78, 352-360.

Erikson, E. (1972). Adolescence et crise : La quête de l'identité. Paris : Flammarion.

Ford, M.E., \& Ford, D.H. (Eds.) (1987). Humans as self-constructing living systems: Putting the framework to work. Hillsdale, NJ: Erlbaum.

Guichard, J. (2004). Se faire soi. L'orientation scolaire et professionnelle, 33, 499-534.

Guichard, J. (2008). Proposition d'un schéma d'entretien constructiviste de conseil en orientation (life designing counseling) pour des adolescents ou de jeunes adultes. L'orientation scolaire et professionnelle, 37(3), 413-440.

Hall, D.T. (1976). Careers in organizations. Glenview, IL: Scott Foresman.

Hall, D.T. (2002). Careers in and out of organizations. Thousand Oaks, CA: Sage. 
Hall, D.T. (2004). The protean career: A quarter-century journey. Journal of Vocational Behavior, 65, 1-13.

Herenberg, A. (1998). La fatigue d'être soi : dépression et société. Paris : Odile Jacob.

Larson, R.W. (2000). Toward a positive psychology of youth development. American Psychologist, 55, 170-183.

Lemoine, C. (2007). De la question à l'auto-questionnement : questionner, source d'emprise et d'appropriation multiple. Psychologie du travail et des organisations, 13(4), 5-24.

Lemoine, C. (2011). Épistémologie induite par le bilan de compétences. In A.M. Vonthron, S. Pohl \& P. Desrumaux (éd.) : Développement des identités, des compétences et des pratiques professionnelles. Paris : L'Harmattan.

Mary, G. \& Costalat-Founeau, A.M. (2012). La dynamique du système capacitaire : Identité, action et projet. Psychologie du travail et des organisations, 18(1), 7-16.

MacMahon, M. (2008). Qualitative career assessment: a higher profile in the $21^{\text {st }}$ century? In J.A. Athanasou \& R. Van Esbroeck (Eds.) International Handbook of Career Guidance, 133-156. New York, NY: Springer.

Mead, G.H. \& Morris, C.W., (1934). Mind, self, \& society: from the standpoint of a social behaviorist. Chicago, IL: University of Chicago Press.

Ninot, G. \& Costalat-Founeau, A.M. (2011). Approche dynamique et temporalité en psychologie sociale. Psychologie française, 56(1), 31-44.

Patton, W. \& McMahon, M. (1997). Career development in practice: A systems theory perspective. Sydney, Australia: New Hobsons Press.

Patton, W. \& McMahon, M. (2006). Career development and systems theory: connecting theory and practice. Rotterdam, NL: Sense Publishers.

Piaget, J. (1936). La naissance de l'intelligence chez l'enfant. Neuchâtel : Delachaux et Niestlé. Sartre, J.P. (1943). L’Être et le néant : Essai d'ontologie phénoménologique. Paris : Gallimard. Sartre, J.P. (1946). L'existentialisme est un humanisme. Paris : Gallimard.

Savickas, M.L., Nota, L., Rossier, J., Dauwalder, J.P., Duarte, M.E., Guichard, J., Soresi, S., Van Esbroeck, R., Van Viannen, A. \& Bigeon, C. (2010). Construire sa vie (life designing) : un paradigme pour l'orientation au XxI ${ }^{\mathrm{e}}$ siècle. L'orientation scolaire et professionnelle, 39(1), 5-39.

Touraine, A. (1995). La formation du sujet. In F. Dubet \& M. Wieviorka (dir.), Penser le sujet : Autour de l'œuvre d'Alain Touraine. Paris : Fayard.

Vygotsky, L.S. (1986, $1^{\text {st }}$ ed. 1936). Thought and language. Cambridge: MIT Press.

Young, R.A. \& Collin, A. (2004). Introduction: Constructivism and social constructivism in the career field. Journal of vocational behavior, 64, 373-388.

Young, R.A. \& Valach, L. (1996). Interpretation and action in career counseling. In M.L. Savickas \& W.B. Walsh (Eds.), Handbook of career counseling theory and practice (pp. 361-376). Palo Alto: Davies-Black.

Young, R.A. \& Valach, L. (2004). The construction of career through goal-directed action. Journal of Vocational Behavior, 64, 499-514.

Young, R.A. \& Valach, L. (2006). La notion de projet en psychologie de l'orientation. L'orientation scolaire et professionnelle, 35(4), 495-509. 
Zavalloni, M. (1971). Cognitive processes and social identity through focused introspection. European journal of social psychology, 1(2), 235-260.

Zavalloni, M. \& Louis-Guérin, C. (1984). Identité sociale et conscience : introduction à l'egoécologie. Montréal : PUM.

Zavalloni, M. (2007). Egoécologie et identité : une approche naturaliste. Paris : Presses Universitaires de France.

Zikic, J. \& Klehe, U.C. (2006). Job loss as a blessing in disguise: The role of career exploration and career planning in predicting reemployment quality. Journal of Vocational Behavior, 69, 391-409.

\section{RÉSUMÉS}

La littérature récente sur l'orientation professionnelle fait état d'un renouvellement épistémologique en direction du constructivisme. Des auteurs pointent du doigt le manque d'instruments qualitatifs dans la recherche et la pratique. Dans ce contexte, le concept d'identité a regagné de l'intérêt. Notre recherche exploratoire a permis d'investiguer les liens entre le projet et l'identité. Nous avons eu recours à la méthode IMIS, auprès de cinq participants engagés dans une démarche de bilan de compétences. Les résultats illustrent la pertinence d'une méthode d'exploration de l'identité dans le processus d'aide à l'élaboration de projets.

Recent literature in career counseling reports an epistemological renewal toward constructivism, some authors pointing at the lack of qualitative instruments in research and practice. In this context the concept of identity regained some interest. Our exploratory research allowed us to investigate the ties between professional project construction and psychosocial identity. We used the MISI method with five participants undergoing career counseling. The results illustrate the pertinence of an interview focused on explorating psychosocial identity in the process of helping to elaborate personal and professional projects.

\section{INDEX}

Keywords : identity dynamics, professional project, social constructivism, ego-ecology Mots-clés : dynamique identitaire, projet professionnel, constructivisme social, ego-écologie

\section{AUTEURS}

\section{GHISLAIN MARY}

est ATER en psychologie sociale, Université Montpellier 3 - Laboratoire Epsylon. Thèmes de recherche : dynamique identitaire, capacités, projet professionnel, acculturation. Contact : Université Paul Valéry, Laboratoire Epsylon, 4 boulevard Henri IV, 34000 Montpellier. Courriel : ghislain.mary@gmail.com

\section{ANNE-MARIE COSTALAT-FOUNEAU}

est Professeure à l'Université de Montpellier 3 - Laboratoire Epsylon. Thèmes de recherche : dynamique identitaire, système capacitaire, action, émotions. Contact : Université Paul Valéry, Laboratoire Epsylon, 4 boulevard Henri IV, 34000 Montpellier. Courriel : annemarie.costalat@univ-montp3.fr 